\title{
Aproximación a los sistemas de calidad en el entorno universitario
}

\section{Amparo Martínez ${ }^{\mathrm{a}}$}

${ }^{a}$ Departamento de Genética. Universidad de Córdoba. Campus Universitario de Rabanales. Carretera de MadridCádiz Km 395.14014-Córdoba. ib2mamaa@uco.es

\begin{abstract}
University students of several science related degrees usually finish their undergraduate and master studies without basic training in quality systems, specifically the ISO / IEC 17025 standard, directly aimed to work in analysis laboratories. This may be due to the intense hours of theoretical and practical classes they must face each semester in which the entire program included in the teaching plan of each subject must be covered. However, it is essential that graduates or masters who access the labour market know the methodology of a quality system most of them will have to deal with during their professional activities. A methodology is proposed in which the students of the last courses of university degrees and masters acquire the necessary competences to work under the ISO / IEC 17025 standard. The activity will be structured in theoretical-practical sessions where students will make decisions regarding measurement equipment based on procedures adapted to the international quality standard ISO / IEC 17025. The methodology and development of the different activities proposed are detailed, as well as objectives that are expected to be achieved.
\end{abstract}

Keywords: Laboratory, ISO/IEC 17025, processes, procedures, calibration.

\section{Resumen}

Los estudiantes universitarios de algunas carreras de ciencias en la mayoría de los casos terminan sus estudios de grado y de máster sin una formación básica en sistemas de calidad, concretamente la norma ISO/IEC 17025, directamente dirigida al trabajo en laboratorios de análisis. Esto puede ser debido a los intensos horarios de clases teóricas y prácticas a los que deben enfrentarse cada cuatrimestre para abarcar todo el programa del plan docente de cada asignatura. Sin embargo, es fundamental que los graduados o másteres que accedan al mercado laboral conozcan la metodología de trabajo en un sistema de calidad en el que la mayoría tendrá que desenvolverse en el desempeño de sus actividades profesionales. Se plantea una metodología en la que el alumnado de los últimos cursos de grados y de másteres universitarios adquiera las competencias necesarias para trabajar bajo la norma ISO/IEC 17025. La actividad se estructurará en sesiones teórico-prácticas en los que el alumnado tomará decisiones acerca de la aptitud de determinados equipos de medida en función de los procedimientos adaptados a la norma internacional de calidad ISO/IEC 17025. Se detalla la metodología y el desarrollo de las actividades, así como los objetivos que se plantean alcanzar.

Palabras clave: Laboratorio, ISO/IEC 17025, procesos, procedimientos, calibración. 


\section{Introducción}

Hoy en día los estudiantes universitarios de carreras de ciencias terminan sus estudios de grado y de máster habiendo adquirido unos amplios y sólidos conocimientos teóricos y prácticos y unas competencias relacionadas con el uso de las TICs, capacidad de diseñar experimentos e interpretar los resultados, el compromiso ético con temas medioambientales y sociales o con la utilización de métodos estadísticos en el análisis de datos entre otros. Poco o nada conocen sobre normas de calidad y los procesos y procedimientos a seguir en un laboratorio acreditado. Cuando estos graduados o másteres acceden al mercado laboral, en la mayoría de los casos lo harán en distintos tipos de laboratorios de análisis, bien sean químicos, biológicos, microbiológicos o clínicos. Los laboratorios de ensayo hacen análisis de muestras y emiten unos resultados. Es fundamental que haya un control de todos los procesos y procedimientos que se emplean en el laboratorio para garantizar que los resultados se corresponden a las muestras, pero también que todos los elementos (técnicos, equipos, etc.) que intervienen en el sistema tienen la competencia necesaria para que el resultado sea objetivo. Uno de los aspectos fundamentales es que los equipos funcionen perfectamente y que sus resultados se correspondan con una realidad analítica, para lo que es necesario mantenerlos, verificarlos y calibrarlos. Todos los procesos y procedimientos por los que se rige el trabajo en un laboratorio están diseñados siguiendo los requisitos de la norma ISO/IEC 17025 y todos los trabajadores deben estar familiarizados con los términos de los mismos y generar los registros oportunos que sirvan para garantizar que la calidad del sistema. Es por todo ello que se considera muy importante la formación de los estudiantes en este asunto, y sería recomendable incluir en los planes docentes actividades enfocadas a la adquisición de las competencias necesarias para diseñar, desarrollar e implementar un sistema de calidad. La introducción de estos conceptos en la formación universitaria podría considerarse una innovación educativa según Esteves (2018) ya que se desarrollarían las prácticas de laboratorio planificadas en una asignatura, pero introduciendo la óptica de la calidad. Entre los resultados que se espera obtener estarían el acercamiento del alumnado a la realidad profesional, interdisciplinariedad o penetración y aceptación tecnológica (López-Martín, 2017).

\section{Objetivo}

Dotar a los estudiantes del conocimiento necesario para desarrollar una profesión en el ámbito de un sistema de calidad mediante el desarrollo de actividades en sesiones teórico-prácticas. En este trabajo se presenta la metodología a seguir en uno de los puntos críticos de un sistema de calidad como es la calibración de los equipos de medida, aunque en distintas sesiones se pueden ir desarrollando otros puntos recogidos en la norma de calidad ISO/IEC 17025.

\section{Desarrollo de la innovación}

\section{Recursos didácticos}

\subsection{Recursos metodológicos}

Se emplea una estrategia expositiva teórica de los contenidos combinada con un aprendizaje basado en la resolución de problemas y prácticas reales de calibración de balanzas.

Para las prácticas se realizarán grupos pequeños de dos o tres miembros debido a la necesidad de manejar equipos delicados que deben de manipularse de forma unipersonal. Se favorece así la participación de todos los alumnos en la práctica y el docente tiene la ocasión de observar no sólo la actitud sino también las aptitudes. De esta forma los alumnos que requieran más tiempo para ejercitarse podrán tener la oportunidad de hacerlo mientras sus compañeros puedan hacer las anotaciones y cálculos necesarios y viceversa.

\subsection{Recursos personales y materiales}

Para el desarrollo de la actividad se requerirán equipos audiovisuales, ordenador con cañón de proyección, libros y normas. Se distribuirán fotocopias con los contenidos en forma de protocolos para los 
estudiantes. Se llevará a cabo en un laboratorio de prácticas dotado de balanzas, pesas calibradas que les proporcionará el profesorado y termómetro y barómetro calibrados con la trazabilidad metrológica de los mismos. Se necesitará también un técnico de laboratorio que apoye con su presencia al personal docente y entre los dos resuelvan los problemas e incidencias que puedan surgir durante la práctica.

\section{Actividades de enseñanza y aprendizaje}

La actividad está orientada a estudiantes de tercer o cuarto curso de grado y a alumnado de máster ya que se necesita una formación mínima en técnicas de laboratorio y conocimientos de matemáticas y estadística. Dada la importante carga de prácticas el número de estudiantes no debe de superar el número de 10-12, dependiendo de la capacidad de los laboratorios de prácticas, para garantizar un aprendizaje adaptado a las necesidades de cada uno. Se impartirán las sesiones teóricas en un aula dotada de medios de proyección audiovisual o en el mismo laboratorio y las prácticas en un laboratorio donde los alumnos podrán interactuar con los equipos que habitualmente hay en un laboratorio de ensayo en técnicas de análisis.

Las actividades de enseñanza y aprendizaje de dividen en tres sesiones con una duración de unos 50 minutos cada una, habiendo sesiones en el aula y en el laboratorio. El aula dispondrá de pantalla para proyectar con medios audiovisuales. El laboratorio de prácticas debe de disponer de sitio suficiente para que cinco o seis grupos de alumnos puedan sentarse en las mesas de trabajo para manipular el material de prácticas. La estrategia metodológica se basará en actividades en el aula en las que se darán unas nociones básicas sobre calidad como una sistemática de trabajo. El profesorado hará sugerencias sobre lo que significa un entorno de calidad para que los participantes sugieran ideas y se evaluará el nivel de conocimientos previos sobre el tema que tienen los estudiantes, así como de su aptitud. Se organizarán en grupos de dos o tres componentes. Se les presentará un cuestionario con valores repetitivos de pesadas de tres balanzas donde se deben deducir los tipos de variación presentes y la fiabilidad de las mismas. No se pide que hagan cálculos, sino que expresen cuál de las tres consideran que es la más fiable y los posibles problemas que pueden tener las otras dos y cómo solucionarlos. El profesorado se hará una idea del entusiasmo por participar en la resolución del problema y la imaginación de cada estudiante para proponer soluciones.

En una segunda sesión se hará una exposición de los contenidos siguientes: principios básicos de calidad, concepto de certificación y acreditación, procedimientos normalizados de trabajo, materiales de referencia y patrones, mantenimiento y verificación de equipos y concepto de incertidumbre y calibración. Los estudiantes deberán elaborar un programa de mantenimiento y calibración de una serie de equipos diferenciando aquellos que son de medida de los que no. Por último, se pondrá a disposición de los estudiantes una balanza, pesas calibradas con su incertidumbre detallada y un ordenador con una hoja de cálculo donde se puedan introducir las pesadas que se vayan realizando. Se harán los cálculos necesarios para expresar la incertidumbre de la balanza. Se debe de informar si a raíz de los resultados obtenidos la balanza es apta o no para su uso.

\section{Evaluación}

\subsection{Aprendizaje del alumno}

Se deben poder evaluar las destrezas adquiridas y habilidades personales y sociales vinculadas a la gestión de equipos de un laboratorio en un entorno de calidad.

\section{Evaluación inicial}

El profesorado evaluará los conocimientos previos, la participación o actitud de cada uno de los alumnos. Una vez terminada la parte práctica se evaluarán los estudiantes mediante un cuestionario y los grupos de alumnos deben razonar las conclusiones que obtienen en cada caso y posibles soluciones o medidas a tomar en cada caso. Se valorará si son capaces de aproximarse a los conceptos de coeficiente de variación y exactitud. Se valorará también si las propuestas de solución son razonables o no. El docente clasificará a los estudiantes en dos tipos en función de sus conocimientos previos y actitud. Con esta información hará grupos mixtos para crear sinergias entre los alumnos. 


\section{Evaluación formativa}

Se hará una autoevaluación de una duración de 10 minutos en la que se plantea la necesidad de realizar análisis sencillos de masa y volumen en un laboratorio teórico. Se indicarán varios protocolos con diferentes ensayos. Los estudiantes deberán indicar las necesidades de equipos para realizar los ensayos citados. Se plantearán cuatro alternativas para elegir una de ellas con el concepto de incertidumbre de un equipo con la tolerancia de un ensayo.

Por otra parte, se realizará una heteroevaluación organizando a los participantes en grupos mixtos según las conclusiones obtenidas por el profesorado en la evaluación inicial. El tiempo para esta prueba será de 20 min. Se ofrecerá una lista de equipos de un laboratorio estándar y los alumnos deberán elaborar un programa y un plan de mantenimiento y verificación de los equipos. Se identificarán aquellos que necesitan ser calibrados elaborando el programa y plan de calibración apropiados.

\section{Evaluación sumativa}

Se realizará un cuestionario de 10 minutos de duración que tendrá la siguiente estructura:

- Se ofrecerá una lista de diez equipos a los estudiantes y deben de identificar aquellos que necesitan calibración. Puntuación máxima: 10.

- Se dispondrá de una lista de diez equipos con su incertidumbre y se establecen las tolerancias para cada uno de ellos en función del método de ensayo. Se deben indicar que equipos son aptos para su uso. Puntuación máxima: 10.

- Seleccionarán los materiales de referencia o patrones calibrados de entre una lista, que serán necesarios para las labores de calibración de una balanza de precisión en un rango de $10 \mathrm{mg}$ a 100 g. Puntuación máxima: 10.

- Harán una ficha de un equipo de medida que se quiera integrar en un sistema de calidad. Puntuación Máxima: 10.

El mínimo de conocimientos se fija en un $50 \%$ en cada apartado obteniéndose una calificación por conocimientos y aptitudes correspondiente a la media de los cuatro. El profesorado hará un registro con la observación continua de cada alumno desde la evaluación inicial, pasando por el comportamiento y actitud durante las exposiciones orales y las prácticas. También valorará su capacidad de trabajar en grupo y la motivación por buscar soluciones a los problemas. La máxima calificación será de 10.

La calificación final será la suma de ambas calificaciones dividida por dos, siempre que en cada una de ellas se haya obtenido una calificación superior a cinco.

\section{Resultados}

En este trabajo se presenta una propuesta de introducir los sistemas de calidad en prácticas de laboratorio de distintas asignaturas de distintos grados universitarios como por ejemplo Genética del grado de Biología, Genética Humana o Ingeniería Genética del grado de Bioquímica. El principal resultado que se espera obtener es el acercamiento de los estudiantes a la realidad profesional con la que se encontrarán al terminar sus grados universitarios. Al final de las sesiones prácticas el alumnado será capaz de alcanzar los siguientes resultados del aprendizaje:

Intelectivos:

- Reconocer las necesidades de equipos de un laboratorio.

- Identificar las medidas apropiadas de mantenimiento, verificación y de los equipos.

- Identificar los equipos de medida que requieren una calibración.

- Calibrar equipos de masa típicos de un laboratorio de análisis biológicos

- Determinar las necesidades específicas de materiales de referencia para usarlos como patrones en los protocolos técnicos.

- Integrar en un sistema de calidad todo lo referente al tratamiento de los equipos y materiales de referencia 


\section{Procedimentales:}

- Redactar planes y programas de mantenimiento, verificación y calibración de los equipos.

- Reconocer los equipos y calibrar aquellos que realizan mediciones.

- Calcular la incertidumbre de la medida de los equipos y trasladarla a las calibraciones de equipos subordinados.

- Manipular las muestras de referencia y equipos de forma que haya trazabilidad entre las mismas y los resultados obtenidos.

Actitudinales:

- Tomar conciencia de la importancia de trabajar en un entorno de calidad.

- Tomar conciencia de la importancia de tener los equipos en perfectas condiciones para que los resultados se correspondan con la realidad de la muestra.

\section{Conclusiones}

El alumnado será capaz de evaluar el estado de los equipos de un laboratorio de ensayo y detectar las necesidades de mantenimiento, verificación y, en su caso, calibración de los mismos. Manipulará de forma correcta las muestras de referencia y equipos y a la vez que tomará conciencia de la importancia del trabajo en un entorno de calidad y buenas prácticas para garantizar unos resultados fiables.

Con actividades como esta los estudiantes aprenderán la importancia trabajar en un sistema de calidad y serán capaces de diseñar y planificar manuales de procesos y procedimientos que sigan las directrices de la norma ISO/IEC 17025. Esto les facilitará la adaptación al entorno laboral en empresas o instituciones.

\section{Referencias}

Esteves, D. (2018). Colaborar para innovar: contribuciones desde un caso portugués para rediseñar la noción de innovación educativa. Revista Educación, Política y Sociedad, 3 (1), 7-30. Disponible en: https://pdfs.semanticscholar.org/fd99/5194246e71262bd3b7636ca2b8492570007f.pdf

López-Martín, R. (2017). Hacia una innovación docente de calidad en la educación superior. Claves para la reflexión. Foro Educacional, 28, 11-28. Disponible en: https://dialnet.unirioja.es/servlet/articulo?codigo=6429501.

Norma ISO/IEC 17025. Requisitos generales para la competencia de los laboratorios de ensayo y de calibración. Ref. ISO/IEC 17025:2017 (ES). https://www.iso.org/obp/ui/\#iso:std:iso-iec:17025:ed-2:v1:es. 